SMASIS2014-7571

\title{
MODELING AND EXPERIMENTATION OF THICKNESS MODE E/M IMPEDANCE AND RAYLEIGH WAVE PROPAGATION FOR PIEZOELECTRIC WAFER ACTIVE SENSORS ON THICK PLATES
}

\author{
Tuncay Kamas \\ Mechanical Engineering \\ University of South Carolina \\ Columbia, SC, USA
}

\author{
Victor Giurgiutiu \\ Mechanical Engineering \\ University of South Carolina \\ Columbia, SC, USA
}

\author{
Bin Lin \\ Mechanical Engineering \\ University of South Carolina \\ Columbia, SC, USA
}

\section{ABSTRACT}

This paper discusses theoretical and experimental analyses of the standing harmonic waves through the electro-mechanical impedance spectroscopy (EMIS) and guided surface acoustic waves (SAW) through the guided wave propagation (GWP) analyses. Both EMIS and GWP analyses have been carried out by utilizing piezoelectric wafer active sensors (PWAS) for in situ structural inspection. PWAS has recently been extensively employed in many applications such as nuclear-structural as well as aero-structural health monitoring and non-destructive evaluations (NDE). EMIS method is utilized for high frequency local modal sensing to determine the dynamic characteristics of PWAS bonded on nuclear-structural component for in-situ ultrasonics. Rayleigh waves a.k.a., SAW, were generated in relatively thick isotropic elastic plates. Rayleigh waves have the property of propagating close to the plate surface, with rapid attenuation with depth. The polarization of Rayleigh waves lies in a plane perpendicular to the surface so that the effective penetration depth is less than a wavelength. Rayleigh waves are a high frequency approximation of the first symmetric $\left(\mathrm{S}_{0}\right)$ and anti-symmetric $\left(\mathrm{A}_{0}\right)$ Lamb wave modes. As the frequency becomes very high the $\mathrm{S}_{0}$ and the $\mathrm{A}_{0}$ wave speeds coalesce, and both have the same value. This value is exactly the Rayleigh wave speed and becomes constant along the frequency.
In the first part of the study, simplified theoretical constrained PWAS-EMIS model is briefly discussed in relatively high frequency range (in $\mathrm{MHz}$ order of magnitude) in terms of thickness mode. Analytical predictive thickness mode impedance simulations of PWAS bonded on plate-like host structures are presented in corresponding with the experiments. For the experimental analyses, PWAS transducers are affixed on isotropic elastic plates such as aluminum plate in relatively high thickness and on a rail I-beam. The extent of the agreement between the experimental and analytical EMIS analyses of PWAS in thickness mode is presented. The study is followed with GWP tests through the pitch-catch method. Rayleigh wave signal packets which are generated in the relatively thick plate and a rail I-beam in high frequency region are assessed along with the experimental thickness mode PWAS-EMIS results. The tuning curve of Rayleigh wave is determined to show the tuning effect of the structure thickness on producing a dominant Rayleigh wave mode. The significant usage of the tuned Rayleigh wave mode is essentially discussed for the applications in the in-situ inspection of relatively thick structures such as nuclear power plant structures. The paper ends with summary, conclusions and suggestions for future work. 


\section{INTRODUCTION}

Piezoelectric wafer active sensor (PWAS) (1) is lightweighted, inexpensive, unobtrusive, minimally intrusive sensor requiring low-power. PWAS is made of piezoelectric ceramic with electric field polarization, $E_{3}$, across the electrodes deposited on both surfaces. It has recently been extensively employed in many applications for structural health monitoring (SHM) and non-destructive evaluations (NDE) (2,3) through guided wave generation and transduction.

Electro-mechanical impedance spectroscopy (EMIS) method has been utilized to determine the local dynamic characteristics of PWAS bonded on a host structure for in-situ ultrasonics (4) such as in the work presented by Sun and Liang $(5,6)$. They utilized the EMIS method for high frequency local modal sensing. The thickness (out-of-plane) mode theory has been developed for piezoelectric crystal and ceramic resonators. Tiersten (7) presented a pioneering work to develop the analytical solution for the thickness vibration of an anisotropic piezoelectric plate. He used the resonator theory with tractionfree $T=0$ boundary conditions at surfaces of a plate. The analytical in-plane impedance for piezoelectric ceramic transducers such as PWAS has been developed by Zagrai and Giurgiutiu (4, 8). One and two dimensional in-plane E/M impedance models for free PWAS and constrained PWAS were derived to model the dynamics of PWAS and substrate structure in terms of EMIS. They assumed the constant electric field, $E_{3}$, to derive the in-plane EMIS. However, the analytical study for thickness mode of PWAS-EMIS has not been fully performed yet. The present work aims to extend the EMIS model of a constrained PWAS at high frequencies (up to $15 \mathrm{MHz}$ ). Kamas et-al (9) adopted the constant electric displacement assumption used in the literature $(10,11)$ and solved the piezoelectric constitutive equations for the thickness mode. Thickness mode EMIS of free PWAS was derived employing axial wave mode dominating in thickness mode and validated using results from coupled field finite element analysis (CF-FEA) and experimental analysis.

Rayleigh wave that resembles to axial wave mode is an elastic wave that propagates close to free surface with as low penetration into the medium as of the order of its wavelength. Rayleigh wave in an isotropic elastic medium are in many cases an appropriate tool for ultrasonic inspection by utilizing the useful property of Rayleigh waves, the propagation speed is independent of frequency (12). Rayleigh wave, the high frequency guided wave mode in isotropic plates, was interpreted as the convergence of the first anti-symmetric $\mathrm{A}_{0}$ and symmetric $S_{0}$ Lamb wave modes by (13). It can be seen in dispersion curves of Lamb wave modes that for large frequency-thickness products, the wave speeds of $A_{0}$ and $S_{0}$ Lamb wave modes coalesce at the wave speed of a Rayleigh wave. In the literature, the Rayleigh wave a.k.a surface acoustic wave or surface guided wave has been utilized for in-situ monitoring of many types of defects in a medium. (13, 14 and
15) used Rayleigh waves for detection of fatigue crack growth and corrosion through wall thickness with theoretical predictions for the thickness loss.

In the current study, one dimensional analytical thickness mode E/M impedance model for PWAS constrained on a host structure is presented. The analytical PWAS-EMIS results in relatively high frequency range are analyzed for clarity of the trend and easy predictability of the experimental impedance spectra and decent agreement between experimental and analytical E/M impedance spectra by relating the dominant Rayleigh wave mode in high frequency band. Therefore, one can obtain more clear impedance spectra in the Rayleigh wave region since Rayleigh wave is a non-dispersive i.e. wave speed is independent from frequency alike axial waves. Thickness of the substrate is also significant parameter to adjust the thickness mode resonance frequency band where Rayleigh wave mode shows up by using the proof-mass concept of the fact that proof-masses shift system resonance towards optimal frequency point. In a recent study, proof-mass PWAS (PMPWAS) resonator is introduced (16) and analytical and numerical simulations are presented for different thickness of proof-masses bonded on PWAS resonators to study the phenomenon of increasing proof-mass height shifts the local system resonance downward.

The current paper also discusses Rayleigh wave tuning curves calculated analytically in terms of strain and measured experimentally in terms of output voltage and the ratio between output to input voltage. The tuning curve of Rayleigh wave is determined to show the tuning effect of the structure thickness on producing a dominant Rayleigh wave mode. The significant usage of the tuned Rayleigh wave mode is essentially discussed for the applications in the in-situ inspection of relatively thick structures such as nuclear power plant walls.

\section{THEORY}

\section{Constrained PWAS-EMIS}

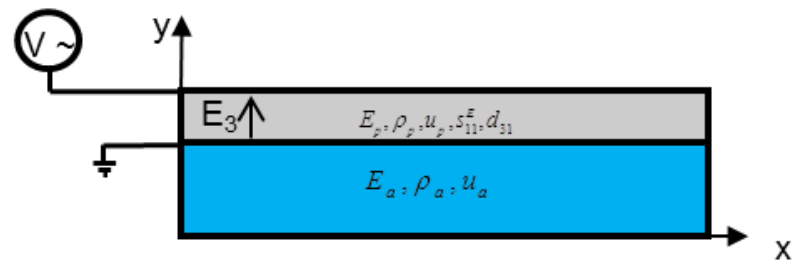

Fig. 1 One dimensional model of a two layer resonator model including a PWAS constrained by an isotropic elastic bar.

The impedance model shown in Eq. (1) for constrained PWAS in thickness mode (16) by using the resonator theory under constrained boundary conditions and constant electrical displacement, $D_{3}$, assumption. The frequency response 
function (FRF) is solved through the normal mode expansion (NME) method and inverse of the FRF gives the dynamic structural stiffness $k_{s t r}(\omega)$ that is substituted into the thickness mode EMIS equation for constrained PWAS by the stiffness ratio, $\quad r(\omega)=k_{\text {str }}(\omega) / k_{P W A S}$ where $k_{P W A S}=A c_{33}^{D} / t$ and $A$ is the PWAS surface area, $c_{33}^{D}$ the stiffness, and $t$ the thickness.

$$
Z=\frac{V}{I}=\frac{1}{i \omega C_{0}}\left[1-\kappa_{33}^{2} \frac{1}{\phi_{t} \cot \phi_{t}+r}\right]
$$

where the E/M impedance is the ratio of $V$ the voltage to $I$ the current, and in terms of $\omega$ the angular frequency, $C_{0}$ the capacitance, $\kappa_{33}$ the E/M coupling coefficient in thickness mode, and $\phi_{t}=0.5 \omega t / c, c=\sqrt{E / \rho}$ is the phase velocity of axial wave, $E$ is the elastic modulus, $\rho$ is the mass density of the host material in which the standing axial wave oscillates,

\section{Rayleigh Wave Tuning Curve}

In this subsection, by assuming 1-D medium, a solution for the tuning curve of Rayleigh wave which resembles to axial wave generated by PWAS ideally bonded on a substrate structure is conducted. The strain as a function of distance on which the axial wave travels is obtained by

$$
\varepsilon(x)=i \varepsilon_{a} \sin \xi_{0} a e^{i\left(\xi_{0} x-\omega t\right)}
$$

for harmonic excitation (17) where $\varepsilon=d u / d x$ the strain that is spatial derivative of the displacement in 1-D medium, $\varepsilon_{a}=d_{31} \hat{E}_{3}$ is the induced strain that is a product of $d_{31}$ the inplane induced strain coefficient and $\hat{E}_{3}$ the amplitude of the induced electrical field between PWAS electrodes, $\xi_{0}=\sqrt{\omega^{2} / c^{2}}$, and $c=\sqrt{E / \rho}$ the phase velocity of axial wave.

Rayleigh wave speed is calculated by

$$
\begin{aligned}
& c_{S}=\sqrt{\frac{E}{2(1+v) \rho}} \\
& c_{R}=c_{S} \frac{(0.87+1.12 v)}{1+v}
\end{aligned}
$$

and the time of flight (ToF) is calculated by

$$
T o F=\frac{D}{c_{R}}
$$

where $D$ is the distance between the transducer and the receiver PWAS bonded on the specimens.

\section{EXPERIMENTAL SETUP}

\section{Constrained PWAS-EMIS Measurement}

The E/M impedance SHM method is direct and convenient to implement, the only required equipment being an electrical impedance analyzer. A HP 4194A impedance analyzer as shown in Fig. 2 was used for the experimental analysis.

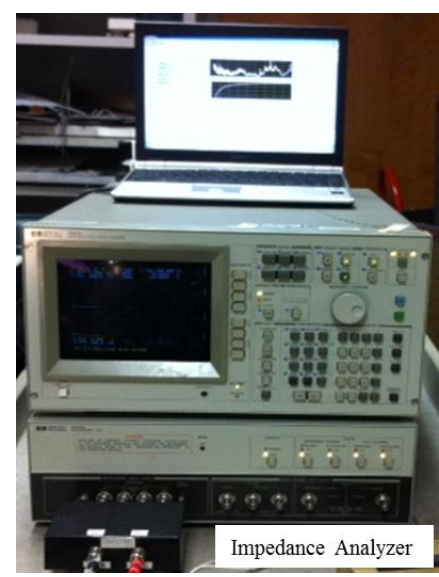

Fig. 2 HP 4194A Impedance Phase-Gain Analyzer.

The impedance analyzer reads the E/M impedance of PWAS itself as well as the in-situ E/M impedance of PWAS attached to a specimen. It is applied by scanning a predetermined frequency range in high frequency band (up to $15 \mathrm{MHz}$ ) and recording the complex impedance spectrum. A LabView data acquisition program was used to control the impedance analyzer and sweep the frequency range in steps that was predefined and to attain the data in a format that assists to data analysis. During the visualization of the frequency sweep, the real part of the E/M impedance, $\operatorname{Re} Z(\omega)$, follows up and down variation as the structural impedance goes through the peaks and valleys of the structural resonances and anti-resonances.

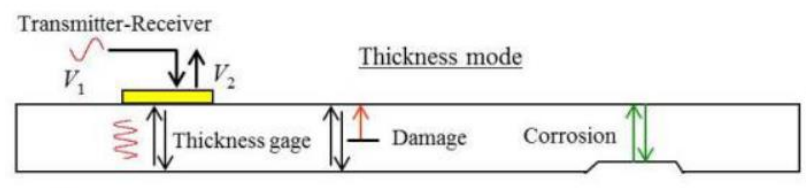

Fig. 3 Schema of thickness mode sensing method (17)

In Fig. 3, PWAS serves as a resonator by both actuating the substrate structure and local modal sensing the standing harmonic waves oscillating in in-plane and out-of-plane (thickness) mode. 


\section{Guided Wave Propagation with Pitch-Catch Method}

In this experimental setup, PWAS transducers serve as high-bandwidth strain sensors for active sensing of far-field. Transmitter PWAS bonded on a substrate structure excites the structure by induced voltage in tone-burst sine wave form with three-counts through the function generator. Then, receiver PWAS senses the wave signals traveling in certain modes along the structure and the received signals as output voltage are read by the oscilloscope in time domain and recorded for postprocessing the data.

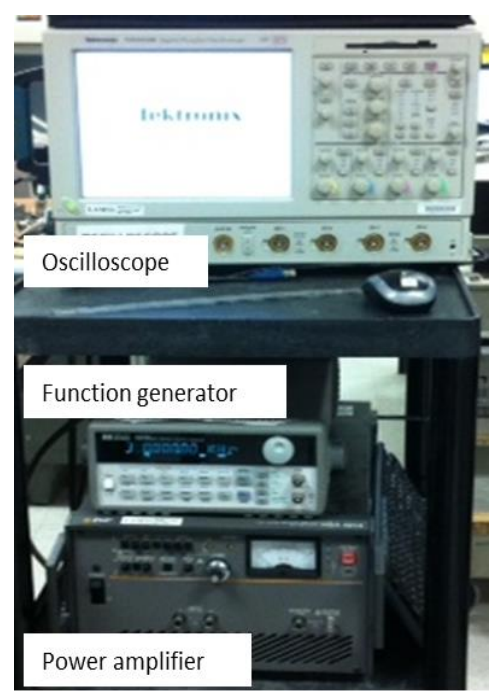

Fig. 4 Tektronix digital phosphor oscilloscope, HP function generator, HSA 4014 power amplifier

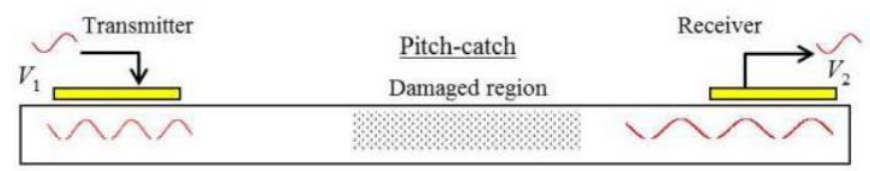

Fig. 5 Schema of pitch-catch sensing method (17)

In the both EMIS and GWP experimental setup, pristine aluminum and steel specimens are used. The first specimen can be seen in Fig. 6. PWAS transducers are bonded on the short edges and clay is applied on both long edges to avoid reflections and obtain more clear signals.

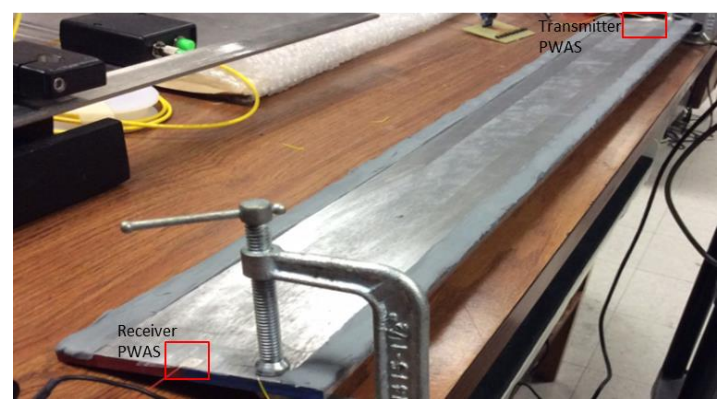

Fig. 6 A pristine aluminum specimen with clay on edges; $1 / 4$ " thick, 4" width, 3' length high strength 2024 aluminum plate

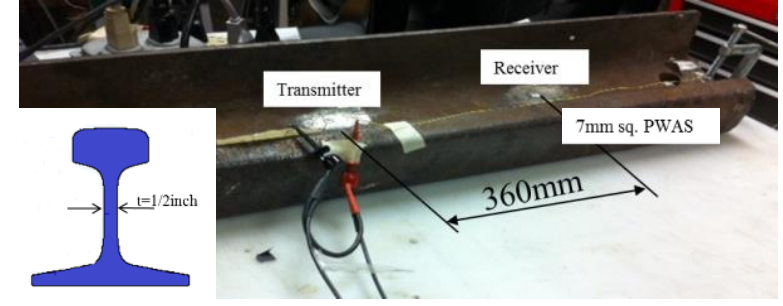

Fig. 7 A pristine steel rail I-beam $1 / 2$ " thick

used for GWP test as another specimen. $7 \mathrm{~mm} \times 7 \mathrm{~mm} \times 0.2 \mathrm{~mm}$ PWAS transducers are bonded on both specimens.

Autotuning GUI (Fig. 8) -developed in LAMSS using LabView software- is utilized to control the function generator and automatically sweep the predefined frequency band and record the data for each frequency step in an excel file then eventually post-process the data to generate the tuning curve for certain wave packets in the received signals.

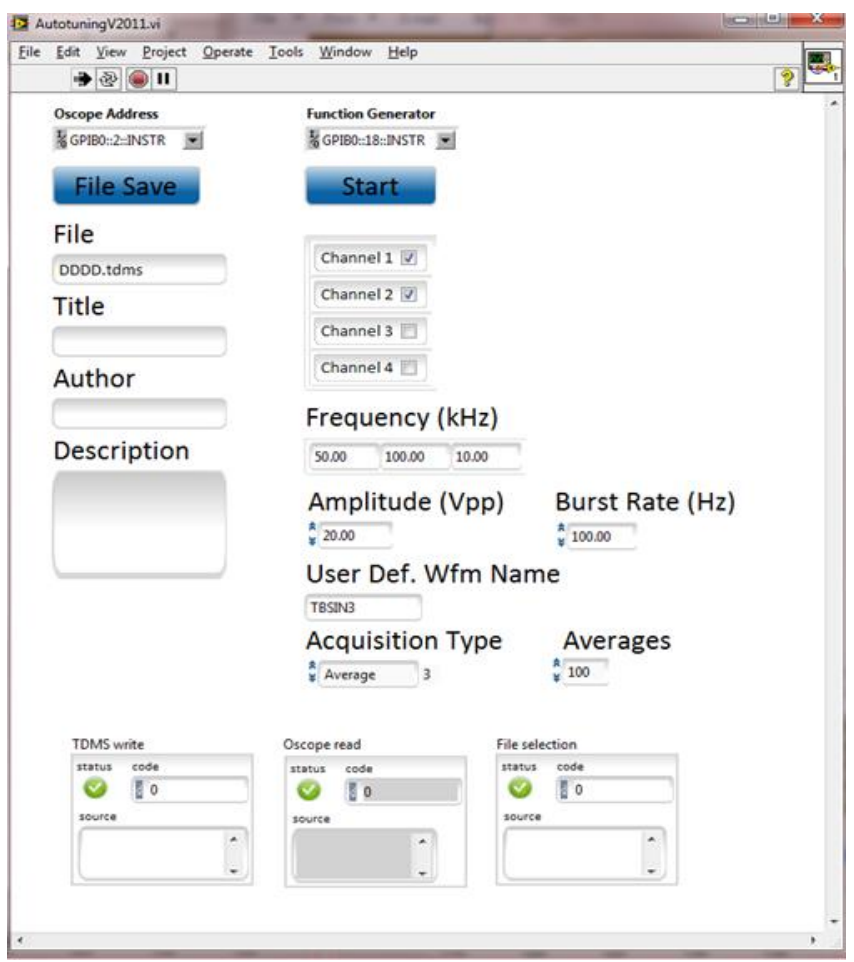

Fig. 8 Autotuning2011 Graphical user interface (GUI), Laboratory for Active Materials and Smart Structures (LAMSS) 


\section{RESULTS AND DISCUSSIONS}

\section{Table 1 Material properties of SM 412 piezoelectric wafer} active sensor

\begin{tabular}{|c|c|c|c|}
\hline PROPERTY & UNIT & SYMBOL & SM412 \\
\hline \multirow{2}{*}{\multicolumn{3}{|c|}{ EQUIVALENCE }} & PZT-5A \\
\hline & & & Navy Type II \\
\hline \multirow{3}{*}{$\begin{array}{l}\text { Electromechanical } \\
\text { Coupling Coefficient }\end{array}$} & & $\mathrm{K}_{\mathrm{p}}$ & 0.63 \\
\hline & & $\mathrm{K}_{\mathrm{t}}$ & 0.42 \\
\hline & & $\mathrm{K}_{31}$ & 0.35 \\
\hline \multirow{3}{*}{ Frequency Constant } & \multirow{3}{*}{ Hz.m } & $\mathrm{N}_{\mathrm{p}}$ & 2080 \\
\hline & & $\mathrm{Nt}$ & 2080 \\
\hline & & $\mathrm{N}_{31}$ & 1560 \\
\hline \multirow{4}{*}{ Piezoelectric Constant } & \multirow{2}{*}{$\mathrm{x} 10^{-12} \mathrm{~m} / \mathrm{V}$} & $d_{33}$ & 450 \\
\hline & & $d_{31}$ & -190 \\
\hline & \multirow{2}{*}{$\mathrm{x} 10^{-3} \mathrm{Vm} / \mathrm{N}$} & $g_{33}$ & 25.6 \\
\hline & & $g_{31}$ & -12.6 \\
\hline \multirow{2}{*}{ Elastic Constant } & \multirow{2}{*}{$x 10^{10} \mathrm{~N} / \mathrm{m}^{2}$} & $\mathrm{Y}_{33}$ & 5.6 \\
\hline & & $\mathrm{Y}_{11}$ & 7.6 \\
\hline $\begin{array}{c}\text { Mechanical Quality } \\
\text { Factor } \\
\end{array}$ & ------- & $\mathrm{Q}_{\mathrm{m}}$ & 100 \\
\hline Dielectric Constant & $@ 1 \mathrm{KHz}$ & $\varepsilon_{\mathrm{T} 33} / \varepsilon_{0}$ & 1850 \\
\hline Dissipation Factor & $\% @ 1 \mathrm{KHz}$ & $\tan \delta$ & 1.2 \\
\hline Curie Temperature & ${ }^{0} \mathrm{C}$ & $\mathrm{T}_{\mathrm{c}}$ & 320 \\
\hline Density & $\mathrm{g} / \mathrm{cm}^{3}$ & $\rho$ & 7.8 \\
\hline
\end{tabular}

A square PWAS in $7 \mathrm{~mm} \times 0.2 \mathrm{~mm}$ dimensions is modeled as a layer on a homogeneous isotropic material (aluminum) substrate layer. The density of the aluminum substrate is 2780 $\mathrm{kg} / \mathrm{m}^{3}$ and the elastic modulus is $72.4 \mathrm{GPa}$. Electromechanical material properties of the piezoelectric transducer are defined in Table 1 .
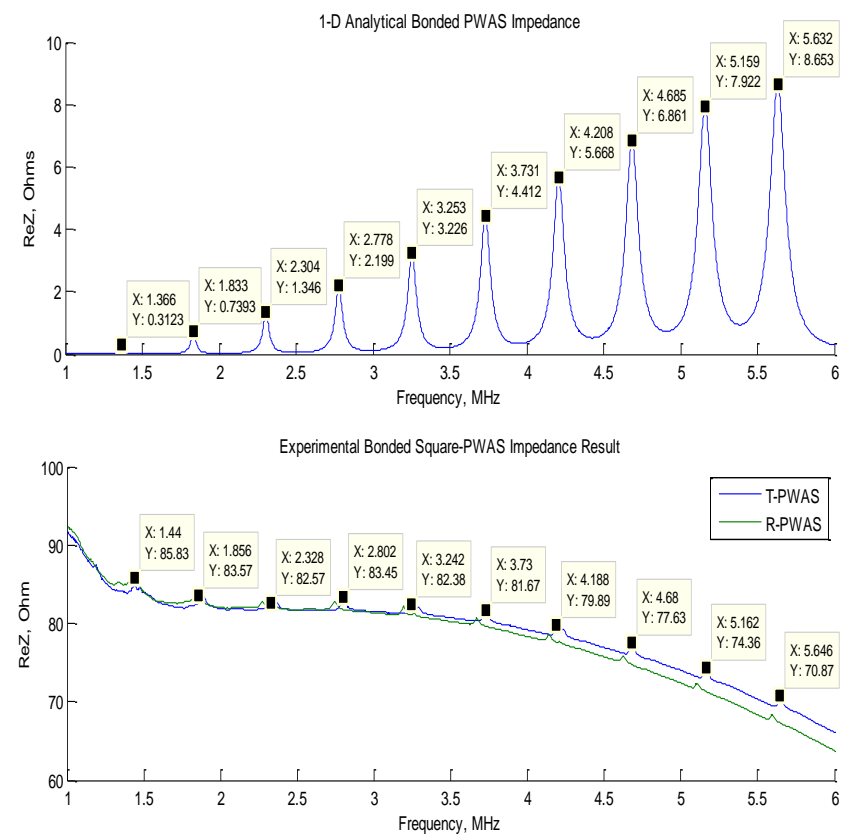

Fig. 9 Analytical and experimental thickness mode EMIS results for $7 \times 7 \mathrm{~mm}^{2}$ square PWAS bonded on 1/4" thick aluminum plate
Two SM412 PWAS are bonded by the two ends of the aluminum specimen (Fig. 6) in distance of $910 \mathrm{~mm}$ to each other. They are first used as a resonator to assess the local dynamic structural signature of PWAS attached on an aluminum substrate by reading its $\mathrm{E} / \mathrm{M}$ impedance spectrum with respect to anti-resonance frequency through the HP 4194A impedance analyzer. The EMIS signature of the specimen of aluminum plate is shown in lower part of Fig. 9 for both PWAS. The EMIS signatures of both PWAS are alike and overlapped. In upper part, the corresponding 1-D analytical prediction of constrained PWAS-EMIS is also shown. The good aggrement in terms of frequency between the analytical and experimental EMIS results is noticeable.

GWP test has also been conducted on the same specimen using the PWAS transducers as transmitter and receiver in this task. Tone-burst sine wave with 3 counts is generated through the function generator to excite the transmitter PWAS and generate a strain wave into the host aluminum plate. The guided wave information travels in the material in different modes and at various wave speeds depending on the excitation frequency-thickness product. In this particular study, we are interested in Rayleigh wave mode, therefore we selected relatively high excitation frequency band to receive the signal dominated by Rayleigh wave modes as can be seen in a few examples of received Rayleigh wave signals (Fig. 10) that travels at constant wave speed i.e. independent from frequency change. The all received wave signals show that Rayleigh wave packet appears distinguishably dominating among other wave packets at the same time window eventhough the frequency increases in the range between $300 \mathrm{kHz}$ and 600 $\mathrm{kHz}$ as seen in Fig. 10. The Rayleigh wave packets appear at even as low frequency as $180 \mathrm{kHz}$ up to $1.8 \mathrm{MHz}$ however the amplitude dramatically decreases after $1 \mathrm{MHz}$ as realized by studying the tuning curve compiled from the experimental data. The tuning curve in the frequency band between 150-600 $\mathrm{kHz}$ is illustrated in Fig. 11. The upper plot shows the analytical calculation of Rayleigh wave tuning curve whereas the lower plot shows the experimental reading of the tuning curve. The trend the analytical and experimental tuning curves agree somewhat closely. They possess the valleys and hills appear in the same frequency bands.

The smooth trends that Rayleigh wave packet draws over frequency and distinguishability of dominating wave packet are promising features that eases predictability and signal processing. As the thicker specimens are analyzed, it's realized that the frequency band where Rayleigh wave packets appear becomes higher. Therefore, it gives the advantage of having the Rayleigh wave mode in also local thickness mode modal sensing because thickness mode EMIS is also for relatively high frequency range in order of $\mathrm{MHz}$. Since Rayleigh wave resembles to the axial wave which has constant wave speed with respect to frequency as seen in the dispersion plot in Fig. 15 , prediction of the E/M impedance signature of the local structure in thickness mode becomes easier. One can use the 
proof-mass concept by increasing the thickness or density of the substrate analyzed to attain Rayleigh wave mode and downshift the local resonance frequency of PWAS-substrate structure so that the thickness mode constrained PWAS-EMIS signature becomes easily predictable by using the standing Rayleigh waves in local structural dynamic sensing
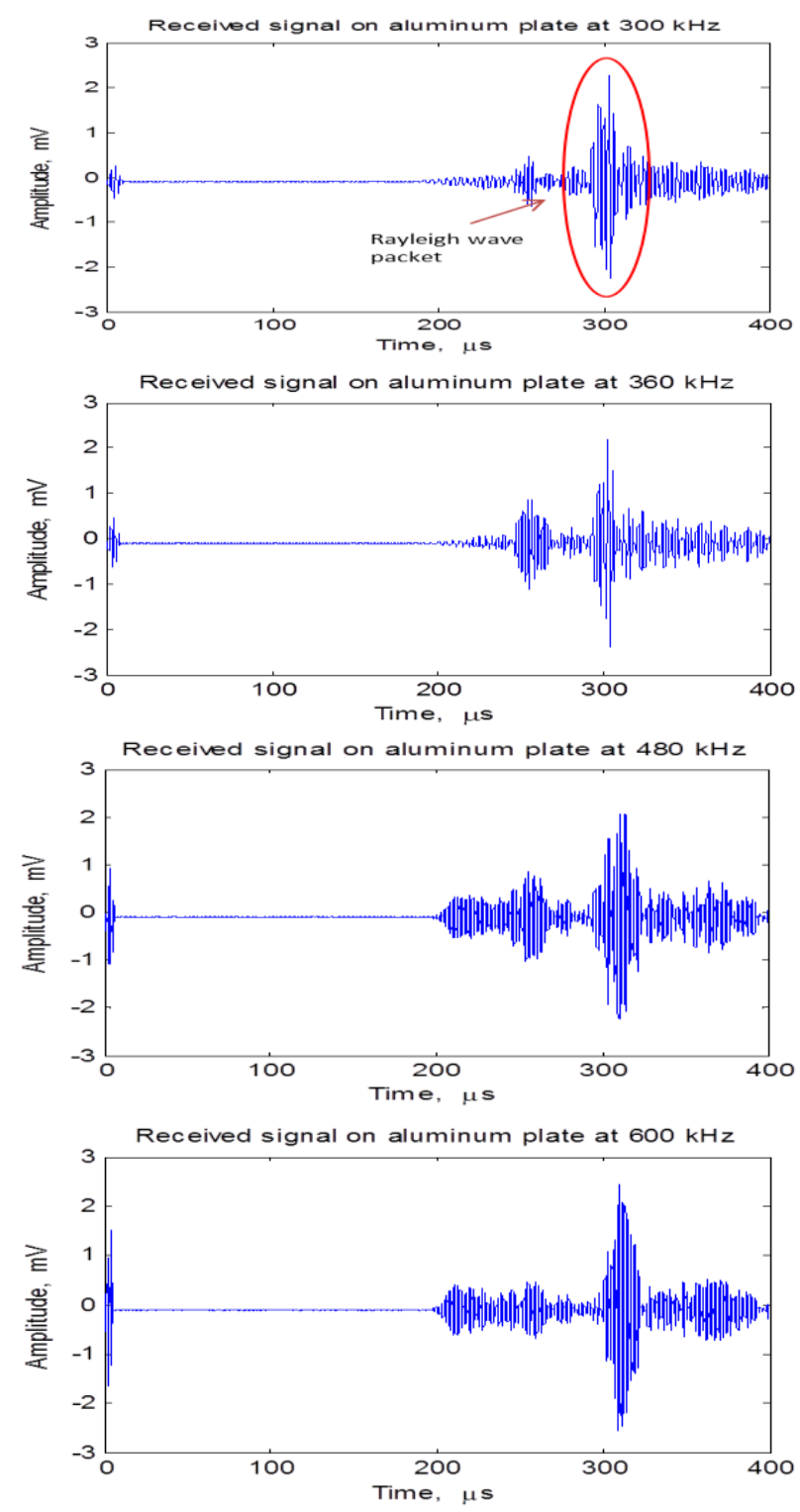

Fig. 10 Received signals from $7 \times 7 \mathrm{~mm}^{2}$ PWAS on 1/4" thick aluminum plate at different frequencies

In Fig. 12, Rayleigh wave phase velocity and time of flight are measured and compared with the calculation by using (3) and (4) respectively. The distance between the trasmitter-PWAS and the receiver-PWAS is $910 \mathrm{~mm}$. The material of the specimen is Aluminum 2024 with the elastic modulus of $72.4 \mathrm{GPa}$, the density of $2780 \mathrm{~kg} / \mathrm{m}^{3}$ and the Poisson's ratio of 0.33 .
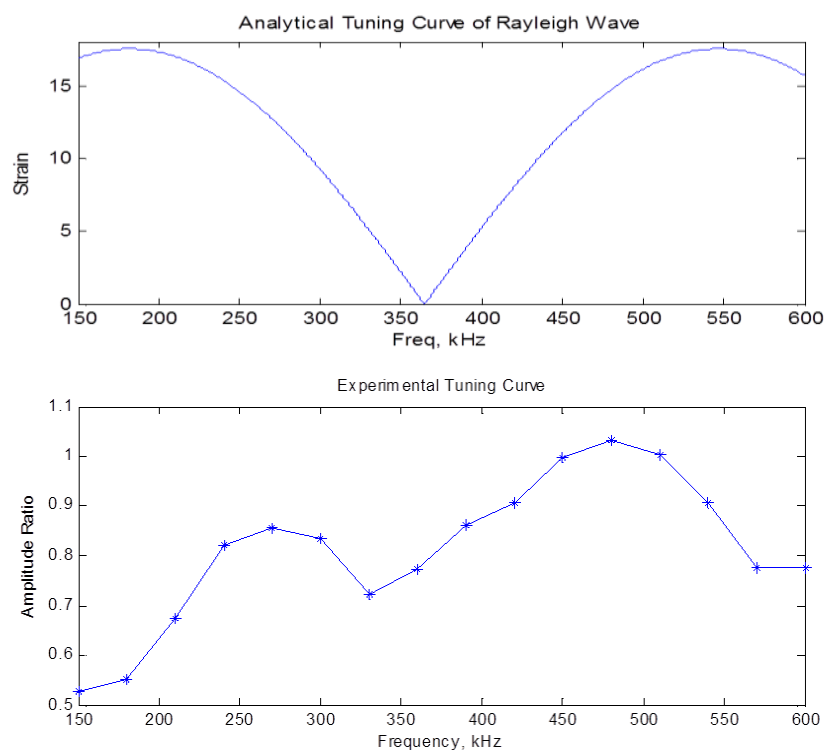

Fig. 11 Analytical and experimental Rayleigh wave tuning curves for $7 \mathrm{~mm} \times 7 \mathrm{~mm} \times 0.2 \mathrm{~mm}$ SM412 PWAS on 6.35 mm thick Aluminum-2024 plate with the elastic modulus of $72.4 \mathrm{GPa}$, the density of $2780 \mathrm{~kg} / \mathrm{m}^{3}$ and the Poisson's ratio of 0.33
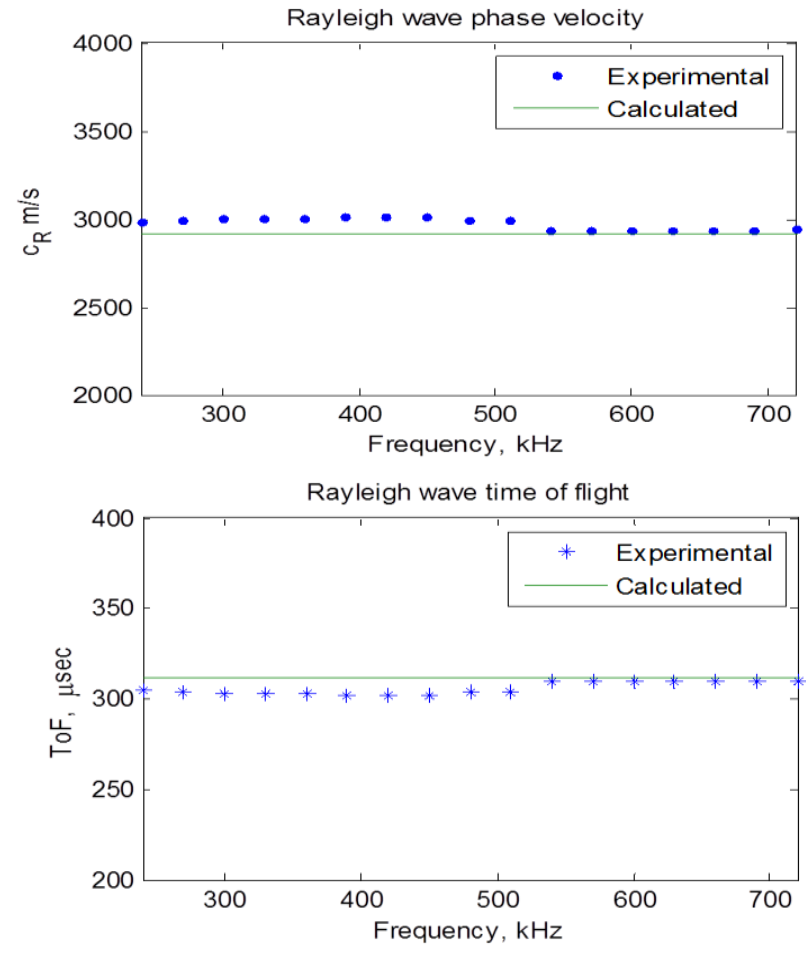

Fig. 12 Rayleigh wave phase velocity and time of flight analytical and experimental results for the distance of $910 \mathrm{~mm}$ between T-PWAS and R-PWAS on $1 / 4$ " thick aluminum plate 

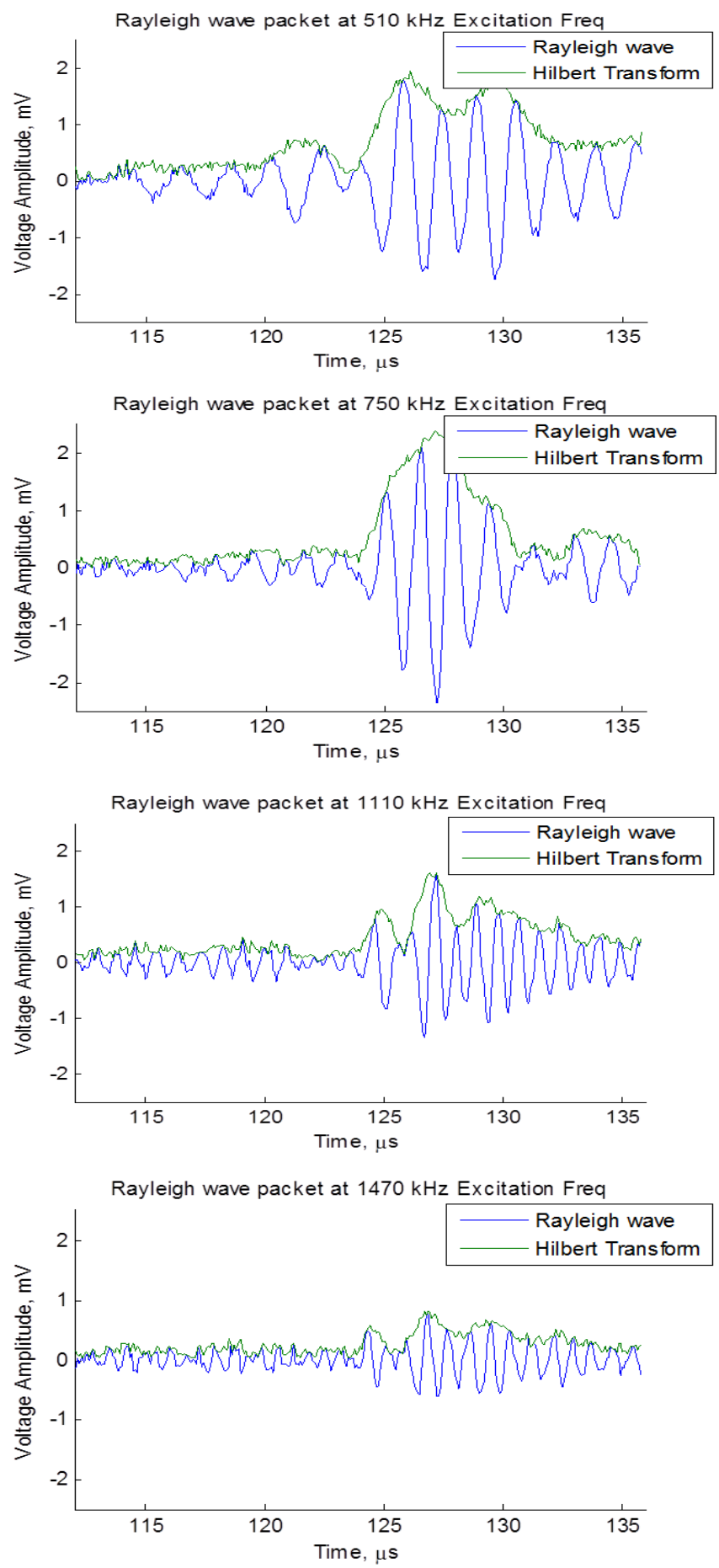

\section{Fig. 13 Received signals from $7 \times 7 \mathrm{~mm}^{2}$ PWAS on $1 / 2$ " thick steel rail I-beam at different frequencies}

Another GWP test is presented on the rail I-beam using the PWAS transducers as transmitter and receiver in distance of $360 \mathrm{~mm}$. Tone-burst sine wave with 3 counts is generated for this test also to excite the transmitter PWAS and generate a strain wave into the host steel rail-beam. Rayleigh wave modes can be seen in a few examples of received Rayleigh wave signals (Fig. 13) that travels at constant wave speed. The all received wave signals show that Rayleigh wave packet appears distinguishably dominating among other wave packets at the same time window eventhough the frequency increases in the range between $510 \mathrm{kHz}$ and $1470 \mathrm{kHz}$ as seen in Fig. 13. The GWP test is conducted up to $1.65 \mathrm{MHz}$ however Rayleigh wave packet amplitude dramatically decreases after $1.5 \mathrm{MHz}$ as realized by studying the tuning curve compiled from the experimental data. The tuning curve in the frequency band between $150-600 \mathrm{kHz}$ is illustrated in Fig. 11. The upper plot shows the analytical calculation of the tuning curve whereas the lower plot shows the experimental reading of the tuning curve. The trends of the analytical and experimental tuning curves agree somewhat closely. They possess the valleys and hills appear in the same frequency bands.
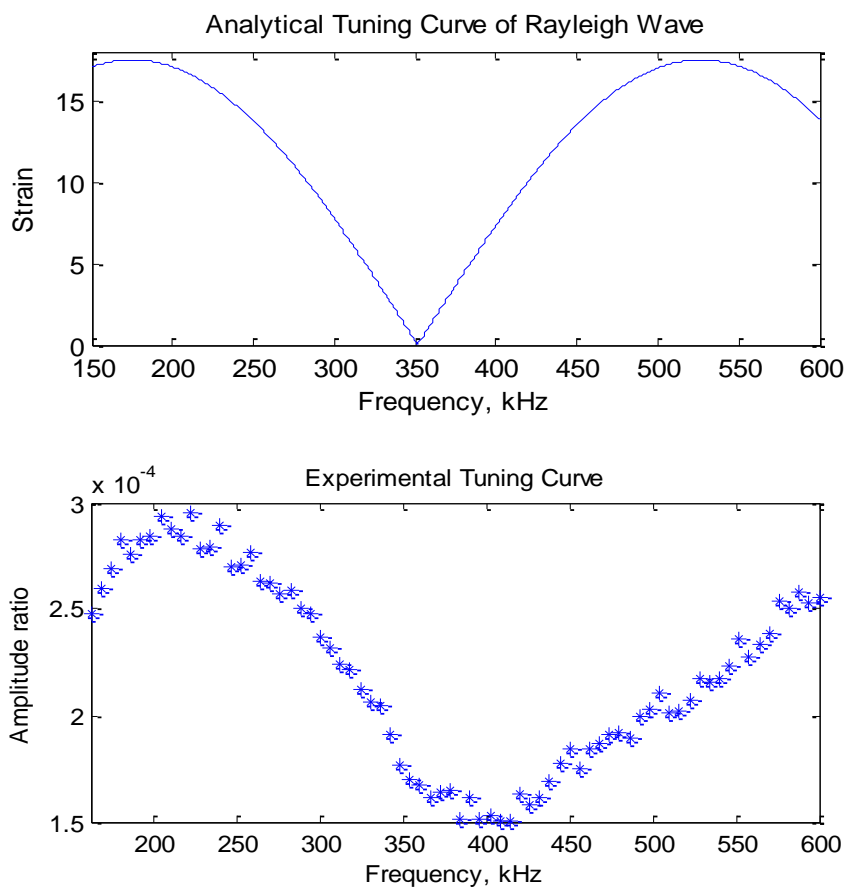

Fig. 14 Analytical and experimental Rayleigh wave tuning curves for $7 \mathrm{~mm} \times 7 \mathrm{~mm} \times 0.2 \mathrm{~mm}$ SM412 PWAS on 12.7 mm thick steel rail I-beam with the elastic modulus of 200GPa, the density of $7850 \mathrm{~kg} / \mathrm{m}^{3}$ and the Poisson's ratio of 0.29

In Fig. 15, Rayleigh wave phase velocity and time of flight are measured and compared with the calculation by using Eqs. (3) and (4) respectively. The distance between the trasmitter-PWAS and the receiver-PWAS is $360 \mathrm{~mm}$. The material of the specimen is Steel-AISI-4340-400F with the elastic modulus of $190 \mathrm{GPa}$, the density of $7850 \mathrm{~kg} / \mathrm{m}^{3}$ and the Poisson's ratio of 0.29 . 


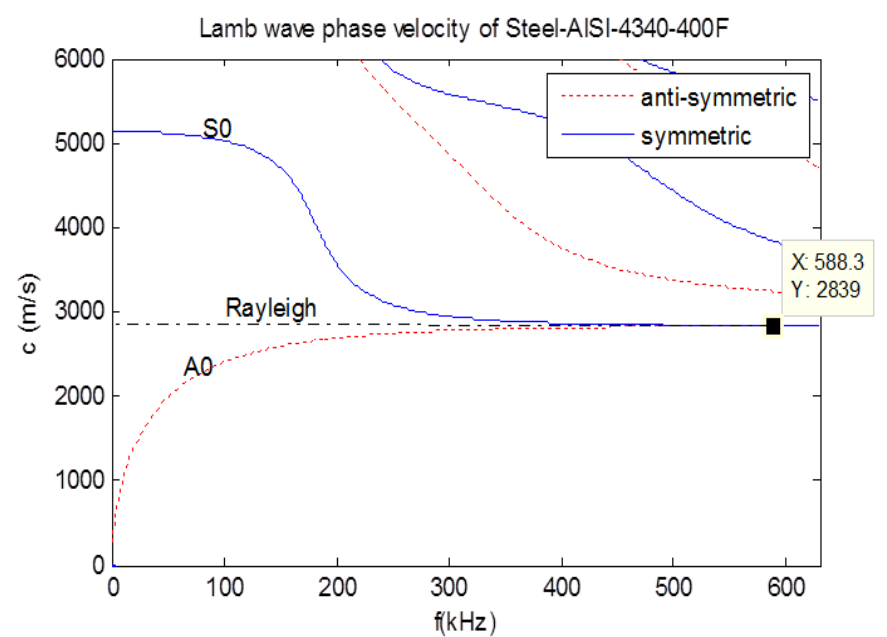

Fig. 15 Dispersion curve that shows Lamb wave mode phase velocities and Rayleigh wave phase velocity at $2839 \mathrm{~m} / \mathrm{s}$
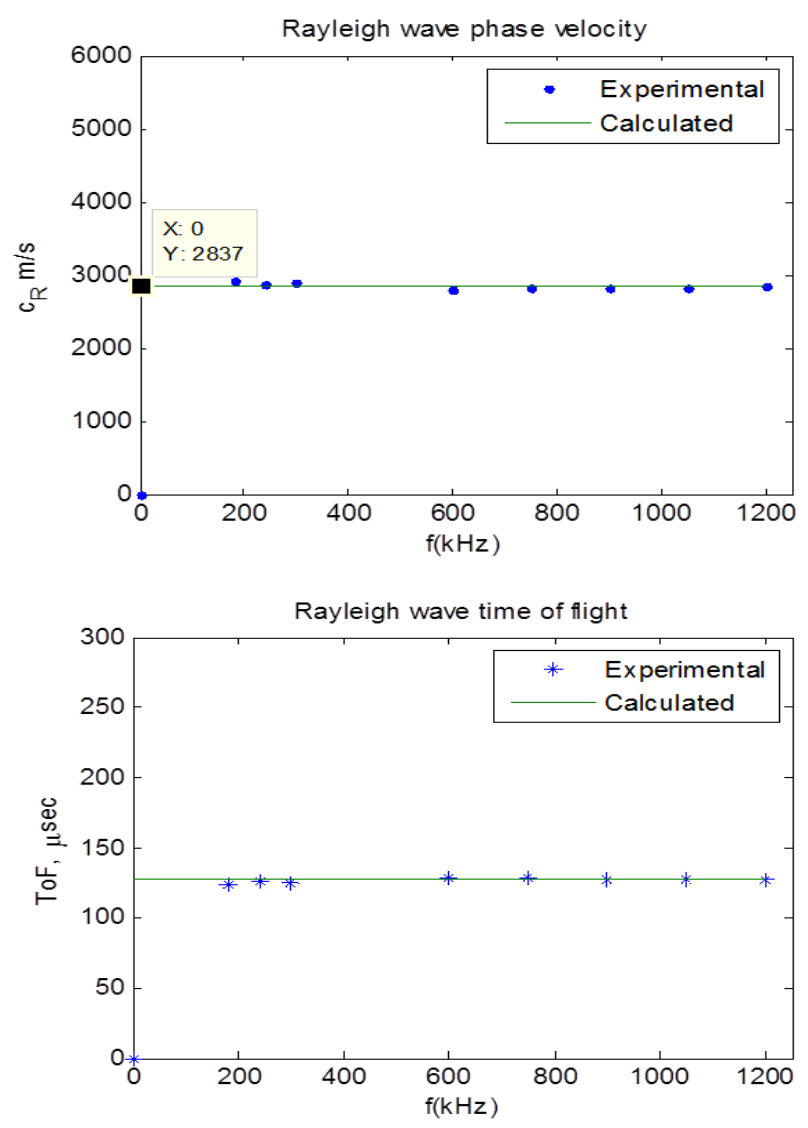

Fig. 16 Rayleigh wave phase velocity and time of flight analytical and experimental results for the distance of $910 \mathrm{~mm}$ between T-PWAS and R-PWAS on $12.7 \mathrm{~mm}$ thick steel rail I-beam

\section{SUMMARY AND CONCLUSIONS}

Electro-mechanical impedance spectroscopy (EMIS) method has been utilized to determine the local dynamic characteristics of PWAS bonded on a host structure for in-situ ultrasonics. Thickness mode EMIS of constrained PWAS was analytically simulated and validated using results from experimental analysis of aluminum specimen.

GWP test has also been conducted on two isotropic elastic specimens such as aluminum and steel by using two SM412 PWAS transducers on each substrate material as transmitter and receiver. In this particular study, we are interested in Rayleigh wave mode, therefore we selected relatively high excitation frequency band to receive the signal dominated by Rayleigh wave modes. Rayleigh wave is elastic wave that propagates close to free surface with as low penetration into the medium as of the order of its wavelength. Rayleigh wave in an isotropic elastic medium are in many cases an appropriate tool for ultrasonic inspection by utilizing the useful property of Rayleigh waves, the propagation speed is independent of frequency

Rayleigh wave trend over frequency and distinguishability of dominating wave packet are promising features that eases predictability and signal processing. The thicker and heavier specimens are analyzed, the higher frequency band where Rayleigh wave packets appear is observed. Therefore, it gives the advantage of having the Rayleigh wave mode as a standing wave in also local thickness mode modal sensing because thickness mode EMIS is also for relatively high frequency range in order of MHz. Since the dominating Rayleigh wave resembles to the axial wave which has constant wave speed with respect to frequency as seen in the dispersion plot in Fig. 15 , prediction of the $\mathrm{E} / \mathrm{M}$ impedance signature of the local structure in thickness mode becomes easier. As seen in the dispersion curves for PWAS ideally bonded on steel substrate, other wave modes such as first symmetric $\left(\mathrm{S}_{0}\right)$ and antisymmetric $\left(\mathrm{A}_{0}\right)$ Lamb wave modes do not interfere as much as Rayleigh wave mode as also obvious in the received signals (Fig. 10 and Fig. 13) in certain frequency band;

One can use the proof-mass concept by increasing the thickness or density of the substrate analyzed to attain Rayleigh wave mode and downshift the local resonance frequency of PWAS-substrate structure so that the thickness mode constrained PWAS-EMIS signature becomes easily predictable by using the standing Rayleigh waves in local structural dynamic sensing 


\section{ACKNOWLEDGMENTS}

Support from National Science Foundation Grant \# CMS0925466; Office of Naval Research \#N00014-11-1-0271, Dr. Ignacio Perez, Program Manager; are thankfully acknowledged.

\section{REFERENCES}

[1] Giurgiutiu, V., Bao, J., and Zhao, W., 2001, “Active Sensor Wave Propagation Health Monitoring of Beam and Plate Structures," Proc of SPIE's 8th International Symposium on Smart Structures and Materials, Newport Beach, CA.

[2] Giurgiutiu, V., and Zagrai, A., 2000, "Damage Detection in Simulated Aging-Aircraft Panels Using The Electro-Mechanical Impedance Technique," Adaptive Structures and Material Systems Symposium, ASME Winter Annual Meeting, Orlando, FL, pp. 1-10.

[3] Giurgiutiu, V., Zagrai, a., and Jing Bao, J., 2002, "Piezoelectric Wafer Embedded Active Sensors for Aging Aircraft Structural Health Monitoring," Structural Health Monitoring, 1(1), pp. 41-61.

[4] Zagrai, A. N., and Giurgiutiu, V., 2001, "ElectroMechanical Impedance Method for Damage Identification in Circular Plates," 40.

[5] Liang, C., Sun, F. P., and Rogers, C. a., 1994, "Coupled Electro-Mechanical Analysis of Adaptive Material Systems -- Determination of the Actuator Power Consumption and System Energy Transfer," Journal of Intelligent Material Systems and Structures, 5(1), pp. 12-20.

[6] Sun, F. P., Liang, C., and Rogers, C. A., 1994, "Structural modal analysis using collocated piezoelectric actuator/sensors: an electromechanical approach,” Proc. SPIE 2190, Smart Structures and Materials 1994: Smart Structures and Intelligent Systems, 238, Orlando, FL.

[7] Tiersten, H. F., 1963, "Thickness Vibrations of Piezoelectric Plates," J. Acoustic Society of America, 35(1), pp. 53-58.

[8] Zagrai, A., and Giurgiutiu, V., 2001, "ElectroMechanical Impedance Method for Crack Detection in
Thin Plates," Journal of Intelligent Material Systems and Structures, 12(October 2001).

[9] Kamas, T., Lin, B., and Giurgiutiu, V., 2013, "Analytical modeling of PWAS in-plane and out-ofplane electromechanical impedance spectroscopy (EMIS)," SPIE Smart Structure and Materials + Nondestructive Evaluation and Health Monitoring 2013, Sensors and Smart Structures Technologies for Civil, Mechanical, and Aerospace Systems, J.P. Lynch, C.-B. Yun, and K.-W. Wang, eds., p. 869227.

[10] Meeker, T. R., 1972, "Thickness mode piezoelectric transducers," Ultrasonics, 10(1), pp. 26-36.

[11] IEEE Ultrasonics, 1987, "IEEE Standard on Piezoelectricity."

[12] Cook, D. A., and Berthelot, Y. H., 2001, "Detection of small surface-breaking fatigue cracks in steel using scattering of Rayleigh waves," 34, pp. 483-492.

[13] Chew, D., and Fromme, P., 2014, "Monitoring of corrosion damage using high-frequency guided ultrasonic waves," 9064 , p. $90642 \mathrm{~F}$.

[14] Fromme, P., 2013, "Noncontact measurement of guided ultrasonic wave scattering for fatigue crack characterization," 8692, p. 86921N.

[15] Masserey, B., and Fromme, P., 2014, "Noncontact monitoring of fatigue crack growth using high frequency guided waves," 9061, p. 90611D.

[16] Kamas, T., Lin, B., and Giurgiutiu, V., 2014, "Modeling and Experimentation of Proof-Mass Piezoelectric Wafer Active Sensor Thickness Mode E/M Impedance Spectroscopy," AIAA Region II Student Conference, Memphis, TN, pp. 1-12.

[17] Giurgiutiu, V., 2008, Structural Health Monitoring with Piezoelectric Wafer Active Sensors, Columbia, SC, USA. 